The content of this document corresponds to the article:

Pellegrini, P., di Tollo, G. \& Pesenti, R. Scheduling ships movements within a canal harbor. Soft Comput (2019). https://doi.org/10.1007/s00500-018-3469-2

Only pagination differs 


\section{Scheduling ships movements within a canal harbor}

Paola Pellegrini, Giacomo di Tollo, Raffaele Pesenti

Paola Pellegrini

Univ. Lille Nord de France, IFSTTAR, COSYS, LEOST, 20, rue Elisee Reclus - BP 70317, F-59666 Villeneuve d'Ascq, France. e-mail: paola.pellegrinieifsttar.fr

Giacomo di Tollo

Department of Management, Ca' Foscari University of Venice, Cannaregio 873, 30121 Venice, Italy. e-mail: giacomo.ditollo@ unive.it

Raffaele Pesenti (corresponding author)

Department of Management, Ca' Foscari University of Venice, Cannaregio 873, 30121 Venice, Italy. e-mail: pesenti@unive. it. Tel: +390412346927. 
Abstract In this paper we propose a model for the in-Port ship Scheduling Problem, that consists in scheduling the movement of ships inside a canal harbor. Our model, which we name RECIP-MILP, is inspired by a model for scheduling trains, to exploit the analogies between a canal harbor and a single track railway network. Moreover, we show how to translate spatial safety constraints into time ones. We apply our model to instances representing ship movements in the Port of Venice. We test the performance of both the exact RECIP-MILP model and a heuristic solution algorithm based on it. We show that we can exactly solve most instances in few minutes.

Key words: in-port ship scheduling problem; ship scheduling; mixed-integer linear programming; Venice.

\section{Introduction}

In this paper, we define a model for scheduling the movements of ships within a canal harbor and we solve it via an exact procedure that respects the physical constraints imposed by canals as well as regulations. The resulting problem is referred to as the in-Port ship Scheduling Problem (PSP). This problem is of interest for port authorities, ship companies, and port operators: they are interested in optimizing the ship movements (and, consequently, ships' waiting time and/or time in port), for several reasons, ranging from consolidating market share and engendering concurrence (Verstichel et al., 2014b; Bugarski et al., 2013) to environmentally related issues (Kontovas and Psaraftis, 2011).

The PSP falls within the rich literature on ship scheduling and routing problems (Christiansen et al., 2013). Specifically, the PSP can be framed within the literature dealing with in-port or canal ship scheduling and routing problems.

Among the in-port problems, the berth allocation and quay crane scheduling problems are amongst the bestknown ones: they aim at determining the mooring berths and scheduling the loading and unloading operations for containerships. The literature presents different recent surveys on the large number of papers on this topic (Bierwirth and Meisel, 2015; Carlo et al., 2015; Gharehgozli et al., 2016). The models introduced by these papers typically consider constraints that take into account different characteristics of container terminals and of ships, such as distance between berths and yards, ship size, and different time constrains or requests of the navigation companies. The berth scheduling problem (see, e.g., Golias et al. (2014)) can also be considered part of the same literature. Other kinds of in-port scheduling and routing problems have been recently proposed in the literature as indicated in the following. The problem of coordinating the movements of tankers that may be required to visit different berths is introduced by Wang et al. (2018). The channel-berth coordination problems are considered in Du et al. (2015), Zhang et al. (2016) and Zhen et al. (2017). In particular, Zhang et al. (2016) define constraints imposing a minimum safety distance between ships sailing a single canal, whereas Zhen et al. (2017) introduce constraints that take into account the variation of the tide level. Furthermore, models for the reduction of gas emissions are proposed by Chang et al. (2013) and Styhre et al. (2017).

The literature on canal problems deals with the scheduling of ships transiting waterways (Ulusçu et al., 2009) or locks (Nauss, 2008; Verstichel et al., 2014a; Passchyn et al., 2016), taking into account different geographical areas, such as the Kiel canal (Lübbecke, 2016), the Yangtze River (Yang et al., 2014; Lalla-Ruiz et al., 2016), the Panama Canal (Franzese et al., 2011), the Istanbul Strait (Ulusçu and Altıok, 2009; Mavrakis and Kontinakis, 2008), and the Welland Canal (Petersen and Taylor, 1988).

Our work is motivated by a collaboration with the Port of Venice, which is a canal harbor inside the Venetian Lagoon. Ships may access the lagoon only through two inlets and may sail only through narrow canals that are dredged within the shallow water. The movement of ships in the lagoon is an important issue for the city of Venice, since it has been shown that it has an impact on the city in terms of both air pollution (Gregoris et al., 2016) and wave generation (Parnell et al., 2015). Indeed, the passage of large cruise ships has given rise to a progressive regulation (Casagrande, 2015). From a topological perspective, the canal layout of the port defines a tree whose leaves are the harbor berths and two roadsteads in front of the lagoon inlets: as canals are narrow, ships cannot cross or give way to one another. To maximize the throughput, movements are usually coordinated in convoys.

Indeed, most of the existing literature considers linear waterways, and is related to specific case-studies. A more general approach is proposed by Lalla-Ruiz et al. (2016), who present a mathematical model and a heuristic 
method to schedule the movements of ships through different waterways in order to minimize the ships' waiting time after their arrival: in their approach, the different waterways are assumed to be independent, and every ship can be assigned to (i.e., can pass through) one waterway only. This approach cannot be applied to the Venitian lagoon, in which the different waterways share some common parts, and other approaches, that consider the peculiarity of the lagoon, have been introduced Canestrelli et al. (2017). In this paper we propose a mixed-integer linear programming (MILP) formulation which effectively models the PSP and allows a realistic representation of the regulations imposed by harbormasters. Differently from Lalla-Ruiz et al. (2016), we assign different weights to positive and negative deviations from the movement scheduled completion times, and we allow to model regulations that impose a safety distance between moving ships: we show how to transform distance safety constraints into time constraints when the canal layout has an articulated structure, and we show that our approach can exactly solve the resulting model in most of the PSP instances of practical interest for the Port of Venice. As pointed out by Canestrelli et al. (2017), the PSP presents some analogies with the problem of scheduling trains on single track networks, and our model, referred as to RECIP-MILP (Research on the Exploitation of Capacity of the Infrastructure of a Port - Mixed-Integer Linear Programming), is inspired to the RECIFE-MILP one, used to solve train scheduling problems (Pellegrini et al., 2015). RECIFE-MILP is a MILP-based heuristic for scheduling and routing trains in real-time which has proven to be effective in different situations, including infrastructures characterized by long single track lines (Quaglietta et al., 2016)

The literature on train timetabling and scheduling is extremely rich (Cacchiani et al., 2014; Lusby et al., 2011), with some studies focusing specifically on single track cases (Nogueira et al., 2015). In this work we exploit the richness of this literature to address the PSP: exploiting the similarities between maritime and rail movements, RECIP-MILP can also be seen as a first step toward the evolution of the management of train movements. Indeed, today's aim in European railway is deploying the European Railway Traffic Management System (ERTMS, see http://www.ertms.net, accessed January 2018). With this deployment, as in the PSP, safety is ensured through the imposition of continuous distances rather than the ones calculated at discrete positions as in the traditional system. Notice that an additional source of complexity of the rail problem is due to the possibility of stopping trains on the track, while it is not possible to stop ships in a canal, and RECIP-MILP may potentially be extended to do so.

The rest of the paper is organized as follows. In Section 2, we describe how to model the harbor waterways and we introduce the main assumptions of the problem. In Section 3, we present how we translate minimum distance safety constraints into time constraints. In Section 4, we introduce the MILP formulation of the RECIP-MILP and, in Section 5, we present our solution algorithm. In Section 6, we discuss a case study. Finally, we draw some conclusions in Section 7.

\section{Port structure and assumptions}

In this section, we first describe how we model a canal harbor. Then, we state the assumptions that we hold true throughout this paper.

A canal harbor is a network of waterways $G=(V, E)$. The vertex set $V$ includes the navigation points, i.e., the points that are of interest for the navigation of ships:

- berths and roadsteads;

- connection points between two waterways or between a berth and a waterway;

- other relevant points where a particular operation, such as a turn or a pilot boarding/disembark, is carried out or some harbormaster constraint holds, e.g., they delimit areas where particular speed limits are imposed.

The edge set $E$ includes the edges $e=(i, j)$ that join pairs of vertexes $i, j \in V$ such that a ship can sail from navigation point $i$ to navigation point $j$ or vice versa without crossing any other navigation point in $V$.

Given a path $p$ on $G$, we use the notation $x \in p$ to mean that $x$ belongs to path $p$ independently of $x$ being a vertex $i \in V$, an edge $e \in E$ of $G$, or even a generic point $P$ internal to an edge. Moreover, we write $p[i, j]$ to indicate a path from $i$ to $j$, where $i$ and $j$ can be either vertexes in $V$ or points internal to edges. With a little abuse of notation, we indicate in the same way both a path and the set of points that belong to the path itself. In particular, we use $p(i, j)$ to mean the set of all the points of path $p[i, j]$ except $i$ and $j$.

Example 1. Let's consider network $G$ in Fig. 1. Vertex 1 represents the roadstead. Vertexes 8, 9, 12, 14, 16, 18 and 20 represent berths. Vertexes $7,11,13,15,17$ and 19 are connection points between one or more berths and a 


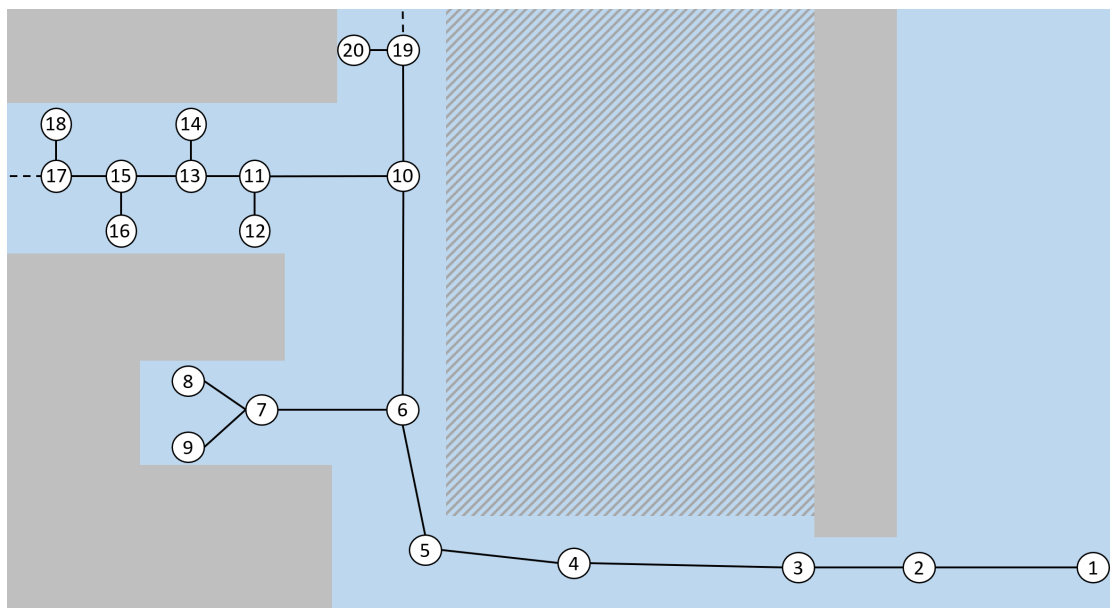

Fig. 1 Example of network $G$ of a port.

waterway. Vertexes 6 and 10 are connection points representing turning basins that connect waterways. Vertexes 2 , 3,4 and 5 are relevant points that delimit areas with particular speed limits. Finally, $p[1,14]$ is a possible path. It represents the route that an entering ship has to follow to reach berth 14 from roadstead 1.

Ships must maintain a minimal safety distance greater than $\ell$ from each other when sailing along the waterways' network. In particular, ships cannot cross or pass each other.

Hereinafter, we use the following notation

- $\mathscr{M}$ : set of movements;

- $r_{m}$ : route of movement $m$;

- $i_{m}, j_{m}$ : starting and final vertexes of route $r_{m}$;

- $s_{m}$ : ship involved in movement $m$;

- $s t_{x, m}$ : sailing time of $s_{m}$ up to vertex/point $x$ on $G$;

- $m h_{m, m^{\prime}}$ : minimum headway time between $m$ and $m^{\prime}$ starting times required to guarantee that $s_{m}$ and $s_{m^{\prime}}$ will maintain a safety distance greater than $\ell$ during their movements;

- $t_{m}$ : time at which $s_{m}$ starts sailing, i.e., leaves the initial vertex of its route;

- $t_{x, m}$ : time at which $s_{m}$ traverses vertex/point $x$ on $G$.

Also, we hold the following assumptions on the topological structure of the network and on the movements of ships:

Assumption 1 A route $r_{m}$ associated to a movement $m$ from berth $i_{m}$ to berth $j_{m}$ is represented by a path $p\left[i_{m}, j_{m}\right]$ of $G$. With a little abuse of terminology we consider the roadstead as a berth.

Assumption 2 The network $G=(V, E)$ is a tree.

Assumption 3 Ships never sail at a speed that is different from the planned one.

Assumption 1 implies that no vertex in a route is visited more than once in a movement. In order to satisfy this assumption, in case a movement requires a ship to sail trough a route that cannot be described by a path we consider the original movement as a series of coupled shorter movements characterized by the path structure.

Example 2. Consider network $G$ in Fig. 1. We can represent the arrival of a ship that sails from the roadstead (vertex 1 ) to the berth in vertex 12 by a single movement along path $p[1,12]$ that visits the vertexes in $\{1,2,3,4,5,6,10,11,12\}$.

Differently, we need two coupled movements to represent the movement of a ship originally moored starboard-side to the berth in vertex 16 that has to moor again starboard-side to the berth in vertex 14 after having reversed its 
direction of travel in the turning basin in vertex 10. Specifically, the first movement occurs along path $p[16,10]$, which includes vertexes $\{16,15,13,11,10\}$, and the second movement occurs along path $p[10,14]$, which includes vertexes $\{10,11,13,14\}$.

Assumptions 2 and 3 allow ensuring that the safety distance between two moving ships is always respected by imposing a minimum headway between the starting times of their operations, as we show in the following section.

Let us now introduce some definitions. Specifically, we say that two points $P$ and $P^{\prime}$ of network $G$ are at a distance $d$ and we write $\operatorname{dist}\left(P, P^{\prime}\right)=d$, if $d$ is the physical distance that a ship has to sail to reach $P^{\prime}$ from $P$ and vice versa. Note that we do not require that either $P$ or $P^{\prime}$ are vertexes of $G$, they may be internal to edges.

Moreover, we say that a point $P$ is at distance $d$ from route $r_{m^{\prime}}$ and we write $\operatorname{dist}\left(P, r_{m^{\prime}}\right)=d$, if $d=$ $\min _{X^{\prime} \in r_{m^{\prime}}}\left\{\operatorname{dist}\left(P, X^{\prime}\right)\right\}$. We say that the distance between two routes $r_{m}$ and $r_{m^{\prime}}$ is $d$ and we write $\operatorname{dist}\left(r_{m}, r_{m^{\prime}}\right)=d$, if $d=\min _{X \in r_{m}, X^{\prime} \in r_{m^{\prime}}}\left\{\operatorname{dist}\left(X, X^{\prime}\right)\right\}$.

Finally, given an ordered set of $s$ points $\left\{P, P^{1}, P^{2}, \ldots, P^{s-1}, P^{s}\right\}$ on $G$, we call circuit the closed walk made by the ordered sequence of the paths $p\left[P, P^{1}\right], p\left[P^{1}, P^{2}\right], \ldots, p\left[P^{s-1}, P^{s}\right], p\left[P^{s}, P\right]$ and we recall that, as $G$ is a tree, any point in the circuit must belong to at least two of the paths (Christofides, 1976).

Example 3. Consider network $G$ in Fig. 1 and two routes $r_{m}$ and $r_{m^{\prime}}$ respectively along path $p[16,20]$ and path $p[8,1]$. The following equalities hold: $\operatorname{dist}\left(r_{m}, r_{m^{\prime}}\right)=\operatorname{dist}(10,6)=\operatorname{dist}\left(10, r_{m^{\prime}}\right)=\operatorname{dist}\left(r_{m}, 6\right)$.

On the other hand, consider the two routes $r_{m}$ and $r_{m^{\prime}}$ respectively along path $p[16,1]$ and path $p[8,20]$. In this case, dist $\left(r_{m}, r_{m^{\prime}}\right)=0$ since all points of edge $(6,10)$ belong to both path $p[16,1]$ and path $p[8,20]$.

Finally, consider the sequences of movements of a ship that sails from the roadstead to the berth in vertex 16 , then moves from this latter berth to the berth in vertex 8 , and hence leaves the harbor reaching again the roadstead. This sequence of movements, which have as extreme points the vertexes in $\{1,16,8\}$, defines a closed walk made by paths $p[1,16], p[16,8]$, and $p[8,1]$.

Now, consider two movements $m$ and $m^{\prime}$. First of all, remark that two routes have distance equal to 0 if they share at least a vertex. Otherwise, as $G$ is a graph-tree, when the distance is greater than 0 there exists one and only one path that joins the two routes (see, e.g., respectively Fig. 2(a) and Fig. 3(a)). The next lemma says that ship $s_{m}$, when sailing along its route $r_{m}$, sees the value of its distance from route $r_{m^{\prime}}$ as a unimodal function of its position and vice versa.

Lemma 1. Consider two movements $m$ and $m^{\prime}$. Let $A \in r_{m}$ be a point at minimal distance from $r_{m^{\prime}}$. Then, the existence of two points $P, Q \in r_{m}$ such that $Q \in p[P, A]$, i.e., $\operatorname{dist}(P, A)>\operatorname{dist}(Q, A)$, implies $\operatorname{dist}\left(P, r_{m^{\prime}}\right) \geq \operatorname{dist}\left(Q, r_{m^{\prime}}\right)$. Symmetrical conditions hold for $r_{m^{\prime}}$.

Proof. We first observe that $A$ exists as the distance function $\operatorname{dist}(.,$.$) is a continuous function defined on a closed$ bounded continuous set of points $p\left[i_{m}, j_{m}\right]$.

Now let $\hat{A}^{\prime}, \hat{P}^{\prime} \in r_{m^{\prime}}$ such that $\hat{A}^{\prime}=\arg \min _{X^{\prime} \in r_{m^{\prime}}}\left\{\operatorname{dist}\left(A, X^{\prime}\right)\right\}, \hat{P}^{\prime}=\arg \min _{X^{\prime} \in r_{m^{\prime}}}\left\{\operatorname{dist}\left(P, X^{\prime}\right)\right\}$, and assume that $\operatorname{dist}\left(P, r_{m^{\prime}}\right)<\operatorname{dist}\left(Q, r_{m^{\prime}}\right)$. Observe that $Q \notin p\left[A, \hat{A}^{\prime}\right]$ by minimality of $A ; Q \notin p\left[P, \hat{P}^{\prime}\right]$ as we are assuming $\operatorname{dist}\left(P, r_{m^{\prime}}\right)<\operatorname{dist}\left(Q, r_{m^{\prime}}\right)$. Finally, $Q \notin p\left[\hat{A}^{\prime}, \hat{P}^{\prime}\right]$ as, again, $\operatorname{dist}\left(Q, r_{m^{\prime}}\right)>\operatorname{dist}\left(P, r_{m^{\prime}}\right) \geq 0$. Then consider the circuit that joins the points $\left\{P, A, \hat{A}^{\prime}, \hat{P}^{\prime}\right\}$. As $Q$ belongs only to $p[P, A]$ we reach the contradictory conclusion that $G$ is not a tree.

As for the respect of the safety distance, if $\operatorname{dist}\left(r_{m}, r_{m^{\prime}}\right)>\ell$ the two ships do not interfere with each other and they can start their operations independently. Otherwise, if dist $\left(r_{m}, r_{m^{\prime}}\right) \leq \ell$ their operations must be coordinated to keep the ships $s_{m}$ and $s_{m^{\prime}}$ always at distance greater than $\ell$. In the next section, we always understand that we are considering two movements $m$ and $m^{\prime}$ such that $\operatorname{dist}\left(r_{m}, r_{m^{\prime}}\right) \leq \ell$.

\section{From distance to time constraints}

In this section, we present the theoretical results ensuring that the safety distance between two sailing ships is always respected by imposing a minimum headway between the starting times of their movements. 
More formally, when considering two movements $m$ and $m^{\prime}$, we have to impose the following condition

$$
t_{P, m} \neq t_{P^{\prime}, m^{\prime}} \quad \Leftrightarrow \quad t_{P, m}>t_{P^{\prime}, m^{\prime}} \quad \text { or } \quad t_{P^{\prime}, m^{\prime}}>t_{P, m} \quad \forall P \in r_{m}, P^{\prime} \in r_{m^{\prime}}: \operatorname{dist}\left(P, P^{\prime}\right) \leq \ell
$$

to ensure that the safety distance between two moving ships is always respected. Indeed, condition (1) prevents $s_{m}$ and $s_{m^{\prime}}$ to be in two points $P$ and $P^{\prime}$ closer than or equal to $\ell$ at the same time.

Theorem 1. Under Assumptions 1-3, the following condition

$$
t_{m}-t_{m^{\prime}}>\max _{P \in r_{m}, P^{\prime} \in r_{m^{\prime}}: d i s t\left(P, P^{\prime}\right) \leq \ell}\left\{s t_{P^{\prime}, m^{\prime}}-s t_{P, m}\right\} \quad \text { or } \quad t_{m^{\prime}}-t_{m}>\max _{P \in r_{m}, P^{\prime} \in r_{m^{\prime}}: d i s t\left(P, P^{\prime}\right) \leq \ell}\left\{s t_{P, m}-s t_{P^{\prime}, m^{\prime}}\right\}
$$

holds if and only if the set of conditions (1) hold.

Proof. To prove the "only if" part of the theorem, we observe that $t_{P, m}=t_{m}+s t_{P, m}$ and that $t_{P^{\prime}, m^{\prime}}=t_{m^{\prime}}+s t_{P^{\prime}, m^{\prime}}$. Hence, we can rewrite (1) as

$$
t_{m}-t_{m^{\prime}}>s t_{P^{\prime}, m^{\prime}}-s t_{P, m} \quad \text { or } \quad t_{m^{\prime}}-t_{m}>s t_{P, m}-s t_{P^{\prime}, m^{\prime}} \quad \forall P \in r_{m}, P^{\prime} \in r_{m^{\prime}}: \operatorname{dist}\left(P, P^{\prime}\right) \leq \ell .
$$

Finally, we note that (2) dominates all conditions (3).

We prove the "if" part of the theorem by contradiction. We assume that condition (2) does not hold even if condition (3) holds, i.e., even if there is no time $t$ such that $\operatorname{dist}\left(X(t), X^{\prime}(t)\right) \leq \ell$ where $X(t)$ and $X^{\prime}(t)$ are the positions of respectively $s_{m}$ and $s_{m^{\prime}}$ in $t$.

To this end, we first observe that condition (2) is equivalent to say that exactly one of the following alternative sets of conditions

$$
\begin{array}{ll}
t_{m}-t_{m^{\prime}}>s t_{P^{\prime}, m^{\prime}}-s t_{P, m} & \forall P \in r_{m}, P^{\prime} \in r_{m^{\prime}}: \operatorname{dist}\left(P, P^{\prime}\right) \leq \ell \\
t_{m^{\prime}}-t_{m}>s t_{P, m}-s t_{P^{\prime}, m^{\prime}} & \forall P \in r_{m}, P^{\prime} \in r_{m^{\prime}}: \operatorname{dist}\left(P, P^{\prime}\right) \leq \ell
\end{array}
$$

must hold. Then we assume by contradiction that two pairs of points $P, Q \in r_{m}$ and $P^{\prime}, Q^{\prime} \in r_{m^{\prime}}$ exist, such that $\operatorname{dist}\left(P, P^{\prime}\right) \leq \ell, \operatorname{dist}\left(Q, Q^{\prime}\right) \leq \ell$ and

$$
\begin{aligned}
& t_{m}-t_{m^{\prime}}>s t_{P^{\prime}, m^{\prime}}-s t_{P, m} \quad \Leftrightarrow \quad t_{m}+s t_{P, m}>t_{m^{\prime}}+s t_{P^{\prime}, m^{\prime}} \\
& t_{m^{\prime}}-t_{m}>s t_{Q, m}-s t_{Q^{\prime}, m^{\prime}} \quad \Leftrightarrow \quad t_{m^{\prime}}+s t_{Q^{\prime}, m^{\prime}}>t_{m}+s t_{Q, m} .
\end{aligned}
$$

Assume without loss of generality that $s_{m}$ reaches $P$ before $Q$, i.e., $s t_{Q, m}>s t_{P, m}$. In this case, we have that

$$
t_{m^{\prime}}+s t_{P^{\prime}, m^{\prime}}<t_{m}+s t_{P, m}<t_{m}+s t_{Q, m}<t_{m^{\prime}}+s t_{Q^{\prime}, m^{\prime}} .
$$

When $s_{m^{\prime}}$ reaches $Q^{\prime}$ before $P^{\prime}$, condition (4) leads to a contradiction as it imposes $s t_{Q^{\prime}, m^{\prime}}>s t_{P^{\prime}, m^{\prime}}$. In the remaining part of the proof, we will show that condition (4) induces a contradiction also when $s_{m^{\prime}}$ reaches $P^{\prime}$ before $Q^{\prime}$. In particular, we will show that if condition (4) holds then there exists a time $t$ such that $\operatorname{dist}\left(X(t), X^{\prime}(t)\right) \leq \ell$.

We preliminarily observe that condition (4) implies that the following sequence of events occurs: first $s_{m^{\prime}}$ reaches $P^{\prime}$, then $s_{m}$ reaches $P$, hence $s_{m}$ reaches $Q$, finally $s_{m^{\prime}}$ reaches $Q^{\prime}$. In other words, when $s_{m}$ is between $P$ and $Q$, we have that $s_{m^{\prime}}$ is between $P^{\prime}$ and $Q^{\prime}$.

This observation allows saying that we have a contradiction if there exists a point $Z^{*} \in p[P, Q] \subseteq r_{m}$ such that $d\left(Z^{*}, Z^{\prime}\right) \leq \ell$ for all $Z^{\prime} \in p\left[P^{\prime}, Q^{\prime}\right] \subseteq r_{m^{\prime}}$.

Next, we will show that this situation occurs when $0<\operatorname{dist}\left(r_{m}, r_{m^{\prime}}\right)<\ell$ and hence $r_{m}$ and $r_{m^{\prime}}$ do not have any point in common. As an example, in Fig. 1, $r_{m}$ could be the route joining the berth in vertex 16 to the berth in vertex 20, while $r_{m^{\prime}}$ could be the route from the roadstead to the berth in vertex 8 .

Let $A \in r_{m}$ be a point at minimal distance from $r_{m^{\prime}}$, respectively $A^{\prime} \in r_{m^{\prime}}$ be a point at minimal distance from $r_{m^{\prime}}$ (in the previous example making reference to Fig. $1, A$ is vertex 10 and $A^{\prime}$ is vertex 6).

Fig. 2 presents all the possible nine different positions, up to symmetry, that points $P, Q, P^{\prime}$ and $Q^{\prime}$ may assume with respect to points $A$ and $A^{\prime}$. We briefly analyze all the different cases. 


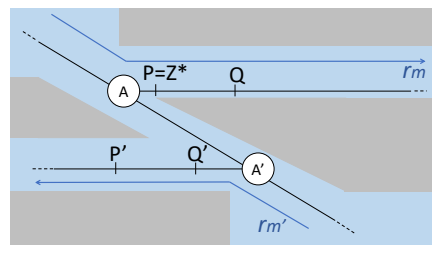

(a)

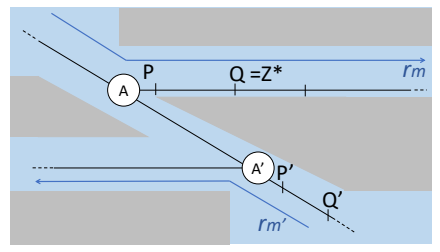

(d)

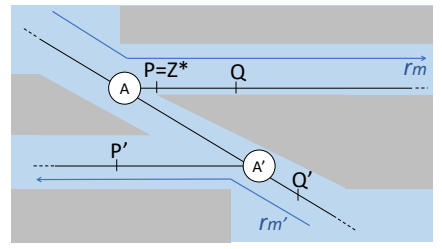

(g)

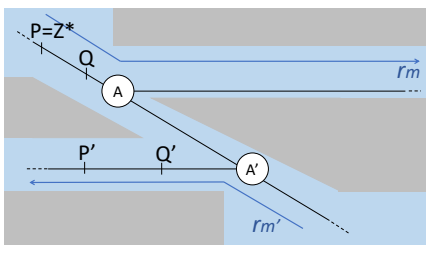

(b)

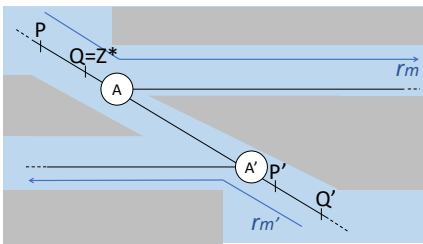

(e)

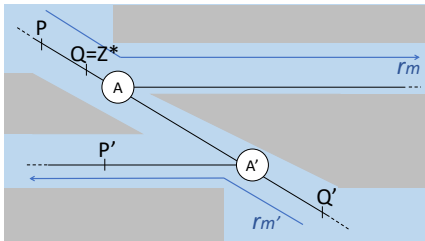

(h)

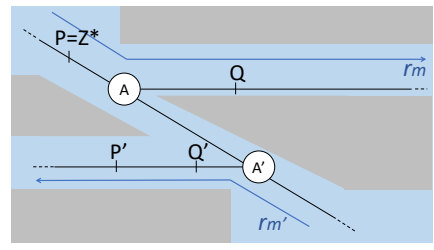

(c)

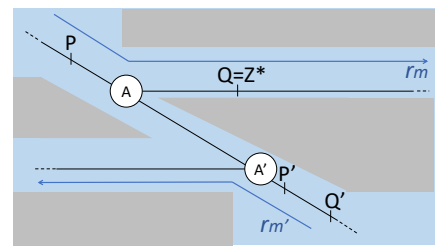

(f)

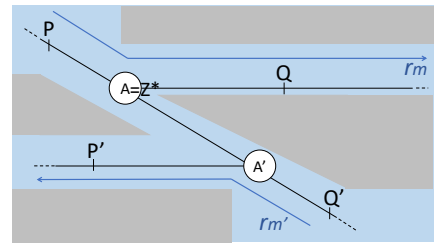

(i)

Fig. 2 Relative position of $p[P, Q]$ and $p\left[P^{\prime}, Q^{\prime}\right]$ when routes $r_{m}$ and $r_{m^{\prime}}$ have no point in common.

In the case in Fig. 2(a), $Z^{*}$ is $P$. Indeed, $d\left(P, P^{\prime}\right) \leq \ell$ by hypothesis. In addition, we have that $p\left[P, P^{\prime}\right] \supseteq p\left[P, Z^{\prime}\right]$ for all $Z^{\prime} \in p\left[P^{\prime}, Q^{\prime}\right]$. Hence, $d\left(P, Z^{\prime}\right) \leq \ell$ for all $Z^{\prime} \in p\left[P^{\prime}, Q^{\prime}\right]$.

Identical argument holds for the cases in Figg. 2(b)-2(c). Symmetric argument holds for the cases in Figg. 2(d)2(f), just swapping point $P$ with point $Q$ and $P^{\prime}$ with $Q^{\prime}$.

In the case in Fig. 2(g), $Z^{*}$ is $P$. Indeed, $d\left(P, P^{\prime}\right) \leq \ell$ and $d\left(P, Q^{\prime}\right) \leq \ell$ as $d\left(P, Q^{\prime}\right) \leq d\left(Q, Q^{\prime}\right) \leq \ell$. In addition, we have that $p\left[P, P^{\prime}\right] \supseteq p\left[P, Z^{\prime}\right]$ for all $Z^{\prime} \in p\left[P^{\prime}, A^{\prime}\right]$ and $p\left[P, Q^{\prime}\right] \supseteq p\left[P, Z^{\prime}\right]$ for all $Z^{\prime} \in p\left[A^{\prime}, Q^{\prime}\right]$. Hence, $d\left(P, Z^{\prime}\right) \leq \ell$ for all $Z^{\prime} \in p\left[P^{\prime}, Q^{\prime}\right]$. Symmetric argument holds for the case in Fig. 2(h).

In the case in Fig. 2(i), $Z^{*}$ is $A$. Indeed, we can observe that $d\left(A, P^{\prime}\right) \leq \ell$ and $d\left(A, Q^{\prime}\right) \leq \ell$. Then, we apply an argument similar to the one used for the case in Fig. 2(g), where we substitute $P$ with $A$.

We now show that we can reach a contradiction also when $\operatorname{dist}\left(r_{m}, r_{m^{\prime}}\right)=0$ and hence $r_{m}$ and $r_{m^{\prime}}$ have at least a point in common. As an example, in Fig. $1, r_{m}$ could be the route joining the berth in vertex 16 to the berth in vertex 9 , while $r_{m^{\prime}}$ could be the route from the roadstead to the berth in vertex 8 . Figure 3 presents all the possible five different positions, up to symmetry, that points $P, Q, P^{\prime}$ and $Q^{\prime}$ may assume. Again, we briefly analyze all the different cases.

In the cases in Fig. 3(a)-3(b), respectively in Fig. 3(c)-3(d), we can prove that $Z^{*}$ is $Q$, respectively that $Z^{*}$ is $P$, by following an argument analogous to the one used for the case in Fig. 2(a).

In the above four situations, we have $p[P, Q] \cap p\left[P^{\prime}, Q^{\prime}\right]=\emptyset$. Then, we finally have to consider the case in Fig. 3(e) where $\operatorname{dist}\left(r_{m}, r_{m^{\prime}}\right)=0$ and $p[P, Q] \cap p\left[P^{\prime}, Q^{\prime}\right]=p[A, B] \neq \emptyset$. In this last case a point $Z^{*}$ may not exists, hence we resort to a different argument.

When $s_{m}$ is in $P$ at time $t_{P}, s_{m^{\prime}}$ must be in $p\left(A, Q^{\prime}\right)$ : indeed, it cannot be in $p\left[P^{\prime}, A\right]$, since $\operatorname{dist}\left(P, Z^{\prime}\right) \leq$ $\operatorname{dist}\left(P, P^{\prime}\right) \leq \ell$ for all $Z^{\prime} \in p\left[P^{\prime}, A\right]$. When $s_{m}$ is in $Q$ at time $t_{Q}$ then $s_{m^{\prime}}$ must be in $p(A, B)$ : indeed, it cannot be in $p\left[B, Q^{\prime}\right]$, since $\operatorname{dist}\left(Q, Z^{\prime}\right) \leq \operatorname{dist}\left(Q, Q^{\prime}\right) \leq \ell$ for all $Z^{\prime} \in p\left[B, Q^{\prime}\right]$. 


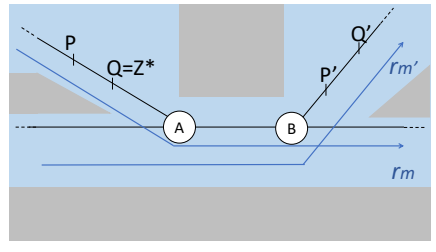

(a)

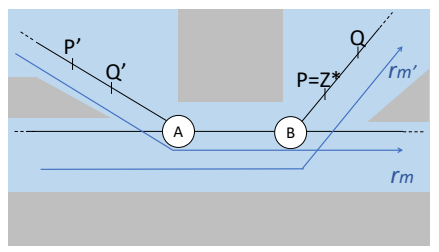

(c)

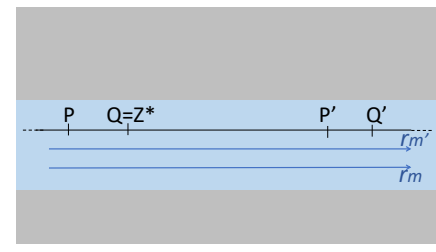

(b)

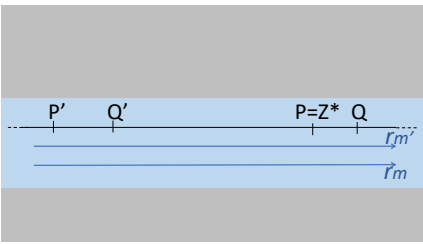

(d)

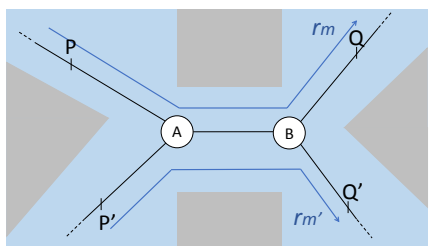

(e)

Fig. 3 Relative position of $p[P, Q]$ and $p\left[P^{\prime}, Q^{\prime}\right]$ when routes $r_{m}$ and $r_{m^{\prime}}$ at least a point in common.

As a consequence $s_{m^{\prime}}$ must be in $p(A, B)$ for all times $t_{P} \leq t \leq t_{Q}$, this means that there exists a time $t^{*}$, with $t_{P} \leq t^{*} \leq t_{Q}$ such that $X\left(t^{*}\right)=X^{\prime}\left(t^{*}\right)$, which is in contradiction with the hypotheses of the theorem.

Hence, the quantities on the r.h.s. of conditions (2) are the infimum time differences which must elapse between the start of $m$ and $m^{\prime}$ to ensure safety, i.e., the infimum headway times.

For any practical purpose, we can then substitute condition (2) with

$$
t_{m^{\prime}}-t_{m} \geq m h_{m, m^{\prime}} \quad \text { or } \quad t_{m}-t_{m^{\prime}} \geq m h_{m^{\prime}, m}
$$

where:

$$
\begin{aligned}
m h_{m^{\prime}, m} & =\max _{P \in r_{m}, P^{\prime} \in r_{m^{\prime}}: \operatorname{dist}\left(P, P^{\prime}\right) \leq \ell}\left\{s t_{P^{\prime}, m^{\prime}}-s t_{P, m}\right\}+\varepsilon \\
m h_{m, m^{\prime}} & =\max _{P \in r_{m}, P^{\prime} \in r_{m^{\prime}}: \operatorname{dist}\left(P, P^{\prime}\right) \leq \ell}\left\{s t_{P, m}-s t_{P^{\prime}, m^{\prime}}\right\}+\varepsilon
\end{aligned}
$$

being $\varepsilon$ a small positive constant. On the other hand, remark that either $m h_{m^{\prime}, m}$ or $m h_{m, m^{\prime}}$ may possibly assume negative values.

\section{RECIP-MILP model}

In this section, we detail the MILP model that we adapt from RECIFE-MILP (Pellegrini et al., 2015) for the ship scheduling problem in a canal harbor. In particular, we use the following notation for any movement $m \in \mathscr{M}$ :

- $w_{m}^{+}, w_{m}^{-}$: weight associated to movement $m$ 's delay, respectively earliness; 
- init $_{m}$ : earliest time at which movement $m$ can start;

- $\operatorname{dead}_{m}$ : latest time at which movement $m$ can start;

- sched $_{m}$ : scheduled time at which movement $m$ should be completed;

- $\mathbb{1}\left(m, m^{\prime}\right)$ : indicator function: 1 if two subsequent movements $m$ and $m^{\prime}$ are operated by the same ship, 0 otherwise;

- $m s_{m, m^{\prime}}, n s_{m, m^{\prime}}$ : minimum, respectively maximum, separation time between the end of a movement $m$ and the start of the subsequent movement $m^{\prime}$ of the same ship, i.e., when $\mathbb{1}\left(m, m^{\prime}\right)=1$;

- $s t_{m}$ : time needed to complete movement $m$;

- $M$ : large constant.

We include the following non-negative continuous variables for all movement $m \in \mathscr{M}$ :

$t_{m}$ : time at which $m$ starts;

$D_{m}^{+}, D_{m}^{-}$: positive, respectively negative, deviation from sched $_{m}$.

In addition, for all pairs of movements $m, m^{\prime} \in \mathscr{M}$ such that $\operatorname{dist}\left(r_{m}, r m^{\prime}\right) \leq \ell$ and $\mathbb{1}\left(m, m^{\prime}\right)=0$, being the index $m$ smaller than the index $m^{\prime}$, we also include the following binary variables:

$$
y_{m, m^{\prime}}= \begin{cases}1 & \text { if } m \text { has precedence over } m^{\prime} \\ 0 & \text { otherwise }\end{cases}
$$

Finally, we formulate our problem as follows.

The objective function to be minimized is the total weighted deviation suffered by movements:

$$
\min \sum_{m \in \mathscr{M}}\left(w_{m}^{+} D_{m}^{+}+w_{m}^{-} D_{m}^{-}\right)
$$

The sets of constraints impose the following conditions:

- a movement $m$ cannot start earlier than init $_{m}$ or later than $\operatorname{dead}_{m}$ :

$$
\text { init }_{m} \leq t_{m} \leq \text { dead }_{m} \quad \forall m \in \mathscr{M}
$$

- the value of deviations $D_{m}^{+}$and $D_{m}^{-}$cannot be less than the difference between the actual and the scheduled times at which movement $m \in \mathscr{M}$ is completed:

$$
\begin{array}{ll}
D_{m}^{+} \geq\left(t_{m}+s t_{m}\right)-\text { sched }_{m} & \forall m \in \mathscr{M}, \\
D_{m}^{-} \geq \operatorname{sched}_{m}-\left(t_{m}+s t_{m}\right) & \forall m \in \mathscr{M} ;
\end{array}
$$

- the separation time between the arrival and departure of movements of the same ship must be between its given minimum and maximum values:

$$
t_{m}+s t_{m}+m s_{m, m^{\prime}} \leq t_{m^{\prime}} \leq t_{m}+s t_{m}+n s_{m, m^{\prime}} \quad \forall m, m^{\prime} \in \mathscr{M}: \mathbb{1}\left(m, m^{\prime}\right)=1 ;
$$

- the start of two movements $m$ and $m^{\prime}$ that can interfere with each other has to be separated by a minimal headway:

$$
\begin{aligned}
t_{m^{\prime}} & \geq t_{m}+m h_{m, m^{\prime}}-M\left(1-y_{m, m^{\prime}}\right) \quad \forall m, m^{\prime} \in \mathscr{M}: \operatorname{dist}\left(r_{m}, r_{m^{\prime}}\right) \leq \ell, \mathbb{1}\left(m, m^{\prime}\right)=0, \\
t_{m} & \geq t_{m^{\prime}}+m h_{m^{\prime}, m}-M y_{m, m^{\prime}} \quad \forall m, m^{\prime} \in \mathscr{M}: \operatorname{dist}\left(r_{m}, r_{m^{\prime}}\right) \leq \ell, \mathbb{1}\left(m, m^{\prime}\right)=0 ;
\end{aligned}
$$

- the arrivals $m$ and $\hat{m}$ and departures $m^{\prime}$ and $\hat{m}^{\prime}$ movements of two different ships at the same berth should not interleave, i.e., the ship that arrives first has to leave before the arrival of the next one:

$$
y_{m, \hat{m}}=y_{m, \hat{m}^{\prime}}=y_{m^{\prime}, \hat{m}}=y_{m^{\prime}, \hat{m}^{\prime}} \quad \forall m, m^{\prime}, \hat{m}, \hat{m}^{\prime} \in \mathscr{M}: \mathbb{1}\left(m, m^{\prime}\right)=1, \mathbb{1}\left(\hat{m}, \hat{m}^{\prime}\right)=1, i_{m^{\prime}}=i_{\hat{m}^{\prime}} .
$$


We can fix part of the $y_{m, m^{\prime}}$ variables during a preprocessing phase. Indeed, condition $y_{m, m^{\prime}}=1$ necessarily holds if $m h_{m, m^{\prime}} \leq i n i t_{m^{\prime}}-$ dead $_{m}$, or if there exists any regulation that imposes that movement $m$ must precede movement $m^{\prime}$, for example due to the type of freight they carry.

\section{RECIP-MILP algorithm}

In this section, we introduce the RECIP-MILP algorithm used to solve the PSP. It is based on the solution of the RECIP-MILP model presented in Section 4.

Two steps are performed. First the we try to solve the RECIP-MILP model using a standard commercial software (XPRESS) for a limited amount of time (five minutes). If an optimal solution is found, the algorithm stops. If at least a feasible solution is found but the optimality proof is not completed, a local search is applied to try to improve the best feasible solution found by the solver. If no feasible solution is found, a constructive heuristic is applied for generating one, which is then passed to the local search for improvement. If the constructive heuristic is not able to identify a feasible solution, the instance is returned as unfeasible.

The local search and the constructive heuristic are based on the following observation. The problem considered is easy once the precedence relations among the movements, that is, the values of the variables $y_{m, m^{\prime}}$, are fixed. On the one hand, the local search procedure consists in iteratively re-optimizing the precedence relations for a different subset $\Gamma \subseteq \mathscr{M}$ of $k$ movements at a time. On the other hand, the constructive heuristic generates its solution iteratively. At each iteration, it considers a different subset $\Gamma \subseteq \mathscr{M}$ of $h$ movements, optimizes the values of their variables $y_{m, m^{\prime}}$ for a limited computational time (one minute) and, finally, fixes the values of these variables for one movement in $\Gamma$.

The sets of $h$ and $k$ movements to be considered at each iteration both in the local search and in the constructive heuristic can be arbitrarily defined. In this work, at each step we consider the first movements in chronological order whose precedence are not fixed yet. The values of $h$ and $k$ are parameters of the algorithm, which need to be set to solve the trade-off between solution quality and computational time.

More formally, the local search procedure applied to a reference solution, coming either from the solution of RECIP-MILP or from the constructive heuristic, can be described as follows:

\section{- Definitions:}

- $\hat{\mathscr{M}}$ is the set of the movements $m$ whose precedence relations are considered definitively fixed;

- $\Gamma$ is the set of $k$ movements whose precedence relations are re-optimized at each iteration;

- restart is a boolean variable indicating that the iterations have to restart;

\section{- Initialization:}

- sort the movements on the basis of precedences determined in the reference solution $z^{\circ}$;

- let $\hat{\mathscr{M}}=\emptyset$;

- set restart $=$ false;

- Iteration:

- let $\Gamma$ include the first $k$ movements $m \in \mathscr{M} \backslash \hat{\mathscr{M}}$;

- solve the relaxed version of the RECIP-MILP formulation where the variables $y_{m, m^{\prime}}$ for $m, m^{\prime} \in \Gamma$ are binary and the remaining ones are set to the values of the reference solution $z^{\circ}$ If the optimal solution of the relaxed problem is not found in one minute, the best feasible solution found is returned;

- if the so obtained solution is better than $z^{\circ}$, then replace $z^{\circ}$ with it. Set restart equal to true;

- set $\hat{\mathscr{M}}=\hat{\mathscr{M}} \cup\{\hat{m}\}$, where $\hat{m}$ is the first movement in chronological order in $\Gamma$;

- if $\hat{\mathscr{M}} \cup \Gamma \neq \mathscr{M}$ iterate; otherwise, if restart is false, stop the iterations as the heuristic has found a local optimum $z^{\circ}$; otherwise, empty the set $\mathscr{M}$ and set restart equal to false, then iterate.

If no feasible solution for REICP-MILP is found within the computational time limit, the constructive heuristic applies the following approach to generate a solution to feed the local search: 
- Definitions:

- $\hat{\mathscr{M}}$ is the set of movements $m$ for which the values of variables $y_{m, m^{\prime}}$ have been set for all $m^{\prime} \in \mathscr{M}$ such that a variable is defined;

- $\Gamma$ is the set of the $h$ movements whose precedence relations are optimized at one iteration;

- Initialization:

- sort the movements on the basis of their expected starting time $\left(s c h e d_{m}-s t_{m}\right)$;

- consider the values of all variables $y_{m, m^{\prime}}$ as not set;

- let $\hat{\mathscr{M}}=\emptyset$;

\section{- Iteration:}

- let $\Gamma$ include the first $h$ movements $m \in \mathscr{M} \backslash \mathscr{M}$ for which there exist some variables $y_{m, m^{\prime}}$ whose values have not been set;

- solve the relaxed version of the RECIP-MILP model where: the variables $y_{m, m^{\prime}}$ already set $\left(m, m^{\prime} \in \hat{\mathscr{M}}\right)$ are considered as data; the variables $y_{m, m^{\prime}}$ for $m, m^{\prime} \in \Gamma$ are binary; the remaining variables $y_{m, m^{\prime}}$ are considered continuous and bounded between 0 and 1 . If the optimal solution of the relaxed problem is not found in one minute, the best feasible solution found is returned;

- if $\hat{\mathscr{M}} \cup \Gamma=\mathscr{M}$, i.e., there are no variables $y_{m, m^{\prime}}$ that are allowed to be continuous, then the optimal solution of the relaxed version of the RECIP-MILP model, if it exists, is a feasible solution for the original problem and the iterations stop;

otherwise, let $\hat{m} \in \Gamma$ be the movement such that the optimal solution of the relaxed version of the RECIPMILP model imposes that $\hat{m}$ has precedence on all the other movements in $\Gamma$, i.e., $y_{\hat{m}, m^{\prime}}=1$ for all $m^{\prime} \in \Gamma$, $\hat{m} \neq m^{\prime}$. Then, set $\hat{\mathscr{M}}=\hat{\mathscr{M}} \cup\{\hat{m}\}$, set $y_{\hat{m}, m^{\prime}}=1$ for all movements $m^{\prime} \in \mathscr{M} \backslash \hat{\mathscr{M}}$ and iterate.

The RECIP-MILP algorithm can be easily adapted for re-scheduling movements in presence of unpredicted events. In the practice, delays in some ship operations or adverse meteo-marine conditions may disrupt part of the planned schedule. Typically, both the earliest times init $_{m}$ and the latest time $\mathrm{dead}_{m}$ at which some movement can start are modified.

In these situations, first port authorities and shipping companies are supposed to negotiate the set $\mathscr{S}$ of movements that need and/or could be considered for re-scheduling. Shipping companies are supposed also to indicate for each of $m \in \mathscr{S}$ whether they desire that the new schedule minimizes the deviation form the original time sched $_{m}$ or from the planned time $t_{m}+s t_{m}$. In the latter case $s c h e d_{m}$ is set equal to $t_{m}+s t_{m}$. Then, the RECIP-MILP algorithm is rerun considering all the values of variables involving the movements in the set $\mathscr{M}=\mathscr{M} \backslash \mathscr{S}$ as a priori fixed and equal to the values that they were assigned in the previous scheduling phase. When no feasible or satisfactory solutions are found the procedure has to iterate. In particular, at each iteration authorities and shipping companies should agree to consider a larger set $\mathscr{S}$ of movement that can be considered for re-scheduling.

Let us conclude this section remarking that in the the prototypical system implemented for the Port of Venice the above solution algorithm is preceded by a preprocessing phase. During this phase the system checks whether the regulations, when applied to the movements in $\mathscr{M}$, induce circular precedences where the sum of the infimum headway times is positive. If this is the case, the PSP instance turns infeasible and the intervention of a human operator is requested.

\section{Computational experiments}

The Port of Venice is a medium-size Italian port (about 3500 calls, for a total 76,000,000 gross tonnage, 610,000 containers - in TEU, and 1,600,000 passengers, with respect to year 2017). Fig. 4 shows the harbor canals, its two inlets (Malamocco and San Nicolò), the sites of 10 passenger terminals in Marittima, and of 23 commercial terminals in Marghera. Fig. 5 reports the distributions of the ship movements in the summer season. Each bar represents the percentage of movements which start in the 60 minutes following the hour indexing the bar itself. It shows that about 13\% of the movements occurs between 6:00 and 7:00 am, most of which are arrival ones. Conversely, about 9\% of the movements occur between 5:00 and 6:00 pm, most of which are departure ones. 


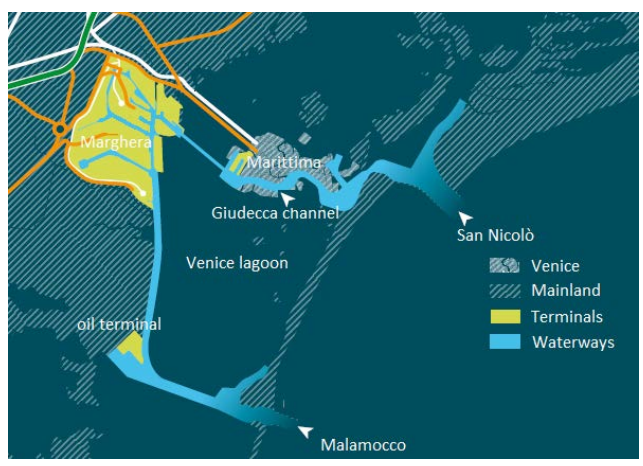

Fig. 4 The port of Venice.

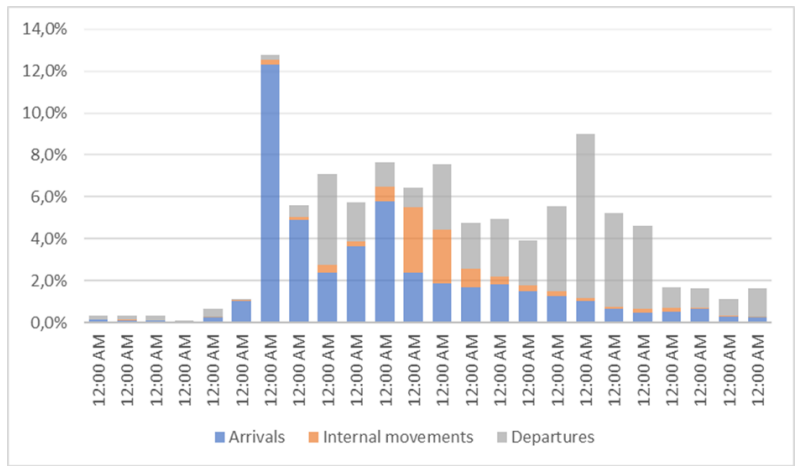

Fig. 5 Distributions of ship movements per hour in the summer season.

The topological layout of the port can be described by a tree with 281 vertexes, among which we distinguish 163 active berths, two roadsteads, 97 junctions, and 19 navigation points, seven of which are the main turning basins.

The computational experiments reported in this section consider the movements occurred during July and August in the years 2015 - 2017, i.e., 183 days. These two months are the most congested ones of the year for this harbor. Specifically, the distribution of ship movements per day in the period of interest is reported in Table 1.

\begin{tabular}{|r|r|}
\hline Number of movements & Number of days \\
\hline less than 10 & 5 \\
\hline between 10 and 19 & 100 \\
\hline between 20 and 29 & 69 \\
\hline 30 or more & 9 \\
\hline
\end{tabular}

Table 1 Distribution of ship movements per day during July and August 2015-2017 in the Port of Venice.

To obtain the instances tackled in the analysis, for each day, we start from the data reporting the actually operated times for each movement (schematized in Fig. 5). We obtain the movements' expected times for our instances by modifying these times. Specifically, we aim to assess the computational time necessary for solving the instances under what are in principle worst case scenarios for the branch and bound procedures used by the solution algorithm presented in Section 5. To do so, we consider instances that may present multiple (almost) equivalent (sub-)optimal solutions, that the procedures manage to cut only at the lowest levels of the branch and bound enumeration tree. We perform three different series of experiments where we round the values of the movements' expected times to the nearest second hour (series A), e.g., 00:00 or 02:00, to the nearest fourth hour (series B), e.g., 00:00 or 04:00, and to the nearest sixth hour (series C), e.g., 00:00 or 06:00. For each of these three series, we solve 183 one-day instances. We consider equal to one, respectively two, the weight associated to the delays, respectively earlinesses, of all the movements $m \in \mathscr{M}$. In addition, we set init $_{m}=0$, dead $_{m}=\infty$. Remark that these choices penalize the 

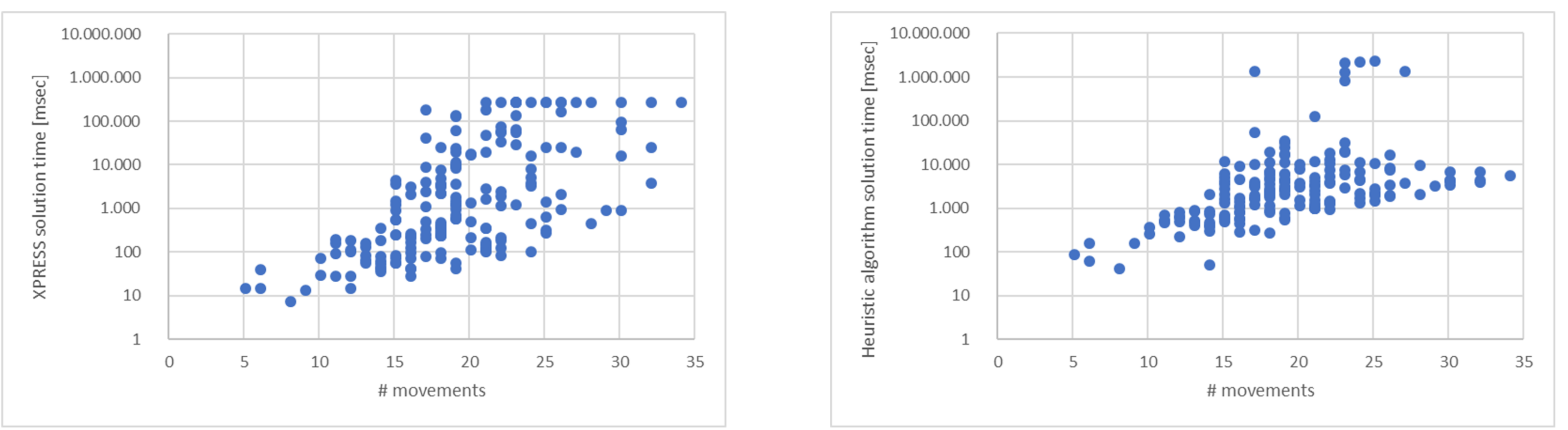

Fig. 6 XPRESS (left) and cumulative constructive heuristic plus local search (right) solution times depending on the size of the instances (y-axis in logarithmic scale)

deviations from the expected times in the same way for all the movements. Then, they also tend to penalize the branch and bound efficiency.

We use XPRESS v.8.2 64 bit as solver. We run the experiments on a laptop PC Dell XPS 15 9550, with an Intel Core i7-6700HQ CPU at $2.60 \mathrm{GHz}$ and $32.0 \mathrm{~GB}$ of installed RAM.

In this experimental analysis, we aim to assess the performance of: i) the exact solution of the RECIP-MILP model found by XPRESS running for a limited computational time; ii) the RECIP-MILP algorithm proposed in Section 5; and iii) the constructive heuristic described in the same section plus the local search procedure, thus simulating the case of the RECIP-MILP model not returning any feasible solution within the time limit.

We set the time limit for the XPRESS solution of the RECIP-MILP model to five minutes. Similarly, in the RECIP-MILP algorithm XPRESS has five minutes to try to solve the model before passing to the constructive heuristic or the local search. In the local search procedure, each evaluation of a neighbor solution of the reference one can last at most one minute. Similarly, in the constructive heuristic, every solution of the relaxed model has this same time limit. Finally, we set $h=10$ and $k=15$. In words, the constructive heuristic considers ten movements in each iteration, and the local search optimizes fifteen movements at a time.

\begin{tabular}{|l|r|r|r|r|}
\hline & \multicolumn{4}{|c|}{ CPU time (ms) } \\
\hline & $5 \mathrm{sec}$ & $30 \mathrm{sec}$ & $1 \mathrm{~min}$ & $5 \mathrm{~min}$ \\
\hline Series A & 173 & 179 & 179 & 183 \\
\hline Series B & 148 & 162 & 166 & 175 \\
\hline Series C & 128 & 149 & 153 & 167 \\
\hline
\end{tabular}

Table 2 Number of instances solved to optimality within different CPU times.

Table 2 reports the number of times in which the exact solution of the RECIP-MILP model is found by XPRESS within different CPU times in the different series of instances. As the table shows, most instances can be solved extremely quickly: for Series A, 95\% of the instances are solved in at most five seconds, and this number decreases to the anyway very good $81 \%$ and $70 \%$ for Series B and C, respectively. Even in the worst scenario for the branch and bound procedures (Series C), the RECIP-MILP model can be solved exactly in the available computational time of five minutes in the large majority of the cases (91\%). In all these cases, the solution of the RECIP-MILP algorithm coincides with the one of XPRESS. Among the 24 instances where this does not happen ( 8 of Series B and 16 of Series C), in six cases the local search procedure manages to improve the solution. For the remaining ones no improvement is achieved, possibly because the solution returned by XPRESS is optimal despite the lack of proof.

The graphs in Fig. 6 describe how the computational times of XPRESS and the heuristic increase as a function of the number of movements in the instances. We show here only instances of Series C as they are the most challenging. As expected, the computational time generally increases with the number of movements, although of course not strictly. What can be observed is that all instances with up to 20 movements can be solved exactly in less than five minutes (left graph): XPRESS stops before this time. Recall that, as shown in Table 1, these instances represent $57 \%$ of the days in the two busiest months of the year. When the instances grow larger, XPRESS still stops 

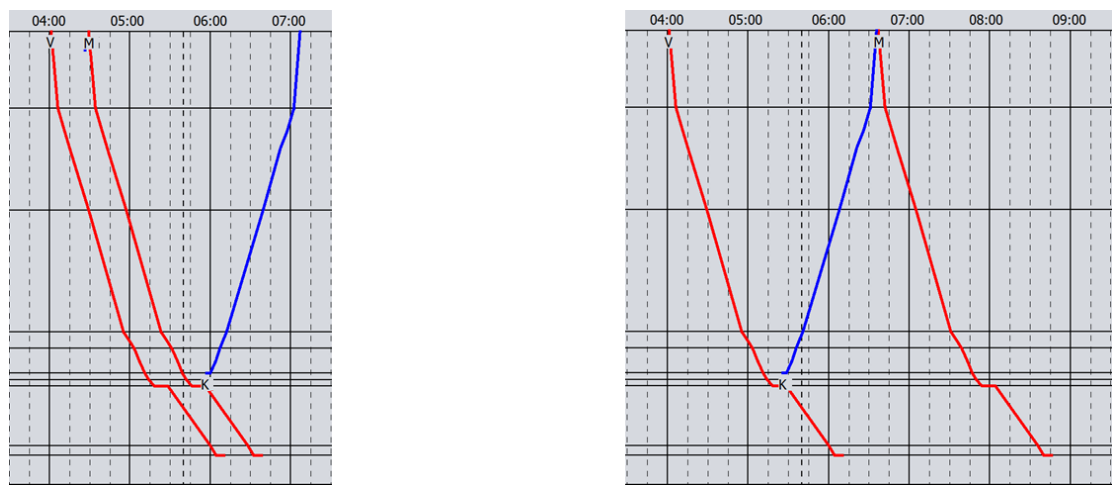

Fig. 7 Example of two different schedules of three movements in a space-time diagram.

before the time limit in the majority of the cases. For 24 instances the use of the RECIP-MILP algorithm becomes important and the switch to the local search phase is performed (not shown in the figure): 6 times out of 24 a better solution was found. In general, the local search procedure requires less than two minutes of computational time but from six cases (not overlapping with the previous ones) in which the computational time increases significantly, reaching at most 25 minutes. The cumulative constructive heuristic plus local search computational times (right graph) are slightly higher than the ones of XPRESS for small instances, and slightly lower for large ones. As an exception, in six instances, the computational time limit of an iteration of the heuristic is saturated, resulting in overall computational times higher than the ones of XPRESS. Finally note for all the instances considered, the computational time requested by the constructive heuristic is less than the 30 seconds.

\begin{tabular}{|r|r|r|r|r|r|r|r|}
\hline \multicolumn{2}{|c|}{} & \multicolumn{2}{|c|}{ Series A } & \multicolumn{2}{c|}{ Series B } & \multicolumn{2}{c|}{ Series C } \\
\hline N. of mov. & N. of inst. & Non-opt. Sol. & Error & Non-opt. Sol. & Error & Non-opt. Sol. & Error \\
\hline $0-19$ & 105 & 0 & & 0 & & 0 & \\
\hline $20-24$ & 51 & 4 & $14 \%-28 \%$ & 9 & $4 \%-26 \%$ & 4 & $1 \%-16 \%$ \\
\hline $25-29$ & 18 & 3 & $11 \%-25 \%$ & 2 & $5 \%-8 \%$ & 3 & $1 \%-20 \%$ \\
\hline 30 or more & 9 & 3 & $10 \%-29 \%$ & 6 & $5 \%-22 \%$ & 7 & $6 \%-26 \%$ \\
\hline
\end{tabular}

Table 3 Number of times in which the two-phase heuristic yielded a value worse than the optimal one and the range of error.

Table 3 reports the number of times in which the constructive heuristic followed by the local search yields a value worse than the one obtained by RECIP-MILP and the range of error. For each series, four classes of instances are considered on the basis of the number of movements involved. These results show that for small instances, the heuristic is always capable of identifying the same solution as RECIP-MILP (the optimal one). Instead, when the number of movements grows, in some cases it fails in doing so. When this happens, the error can be rather large for some instances, reaching almost $30 \%$ of the objective function value in the worst cases. This sometimes bad behavior is justified by the fact that canals in the Port of Venice are quite long, and ships generally require more than an hour to sail them. Consequently, even small differences in the sequence of the movements, and hence, in the times in which a switch in the direction of the ships' flow along a canal occurs, may have significant repercussions on the timing of the movements. To better understand this phenomenon, consider the example in Fig. 7. The figure reports a space-time diagram inspired to what typically done to represent movements in the railway system. The xaxis represents time, while the $y$-axis indicates space. Here, the main canal between Marghera and the Malamocco inlet is represented. Three ships need to travel along this canal, $V, M$ and $K$, and they all desire to start their movements (arrivals in the case of $V$ and $M$, departure in the case of $K$ ) at 4:00 am. Suppose that we can delay the movements but no advance is possible. The diagram on the left side of Fig. 7 shows the optimal schedule: movement $V$ is about 30 minutes late and movement $K$ is about 115 minutes late. On the other hand, the diagram on the right side of the same figure shows the consequences of a switch in the direction of the ships' flow along the canals, i.e., the swapping of the movements $V$ and $K$. In this case movement $K$ is about 80 minutes late and movement $M$ is about 160 minutes late. This unique exchange of sequence in an instance implies then a difference of 90 minutes in the deviations considered by the objective function value. Given the partially greedy nature of the constructive heuristic, this kind of exchanges may well happen in the solution procedure. Hence, when the heuristic is unable to find the optimal solution, only few mistakes may bring it quite far from the optimal value. 


\section{Conclusions}

In this work, we introduced RECIP-MILP, an algorithm based on the solution of a MILP model that allows determining the exact solution of the in-Port ship Scheduling Problem in most of the cases of practical interest for a medium-size canal port. This algorithm may be used to support the user in planning and in quickly re-scheduling the ship movements in presence of unpredicted events that may disrupt the original schedule. We also presented how to translate minimum distance safety constraints into time constrains so that RECIP-MILP can deal with only time-related decision variables.

In the experimental analysis on instances representing ship movements in the Port of Venice, we showed that RECIP-MILP can return the optimal solutions by using a commercial MILP solver, in most of the cases, in no more than a few minutes. To deal with possible more difficult instances, RECIP-MILP includes a heuristic approach based on the solution of the same MILP model iteratively applied to smaller instances. Although in our analysis this approach did not prove to be necessary, we think that it may be useful in more articulated situations. Indeed, it always finds feasible solutions to the instances, and in many cases not far from the optimal one. Moreover, it will suffer less than exact solution in RECIP-MILP from the increase of the size of the branch and bound tree in difficult instances.

RECIP-MILP is the base algorithm we consider in our current research. This research aims at developing a system capable of automatically reformulating a mixed integer programming model for the PSP in presence of different kinds of frequently changing harbormaster's regulations.

\section{Acknowledgments}

The authors want to thanks the North Adriatic Sea Port Authority for its support and suggestion. In particular, Eng. P. Menegazzo and Capt. A. Revedin.

\section{Compliance with Ethical Standards}

This study was partially funded by the "Smart PORt Terminals - SPORT" MIUR-PRIN project (grant number: 2015XAPRKF) financed by the Italian state and by the "CPER ELSAT2020" project co-financed by the European Union with the European Regional Development Fund, the French state and the Hauts-de-France Region Council. The study was partially developed within the Centro Studi su Economia e Management della Portualità of Università Ca' Foscari, Venezia.

Conflict of Interest: Author R. Pesenti has received research funds from the North Adriatic Sea Port Authority and declares that the Centro Studi su Economia e Management della Portualità is partially funded by the North Adriatic Sea Port Authority. Author G. di Tollo declares that he has no conflict of interest. Author P. Pellegrini declares that she has no conflict of interest.

This article does not contain any studies with human participants or animals performed by any of the authors.

\section{References}

C. Bierwirth and F. Meisel. A follow-up survey of berth allocation and quay crane scheduling problems in container terminals. European Journal of Operational Research, 244(3):675 - 689, 2015.

V. Bugarski, T. Backalic, and U. Kuzmanov. Fuzzy decision support system for ship lock control. Expert Systems with Applications, 40:3953-3960, 2013. 
V. Cacchiani, D. Huisman, M. Kidd, L. Kroon, P. Toth, L. Veelenturf, and J. Wagenaar. An overview of recovery models and algorithms for real-time railway rescheduling. Transportation Research Part B: Methodological, 63:15-37, 2014.

E. Canestrelli, M. Corazza, G. De Nadai, and R. Pesenti. Managing the ship movements in the port of venice. Networks and Spatial Economics, 17(3):861-887, 2017.

H. J. Carlo, I. F. A. Vis, and K. J. Roodbergen. Seaside operations in container terminals: literature overview, trends, and research directions. Flexible Services and Manufacturing Journal, 27(2):224-262, Sep 2015. ISSN 1936-6590.

M. Casagrande. The Progressive Regulation of the Passage of Large Cruise Ships in Venice: The Decision-Making Proceedings Between Law and Stakeholder Pressure, pages 185-195. Springer International Publishing, Cham, 2015. ISBN 978-3-319-11385-2.

Y. T. Chang, Y. Song, and Y. Roh. Assessing greenhouse gas emissions from port vessel operations at the Port of Incheon. Transportation Research Part D: Transport and Environment, 25:1-4, 2013.

M. Christiansen, K. Fagerholt, B. Nygreen, and D. Ronen. Ship routing and scheduling in the new millennium. European Journal of Operational Research, 228:467-483, 2013.

N. Christofides. Worst-case analysis of a new heuristic for the travelling salesman problem. Technical Report 388, Graduate School of Industrial Administration, Carnegie Mellon University, 1976.

Y. Du, Q. Chen, J.S. L. Lam, Y. Xu, and J.X. Cao. Modeling the impacts of tides and the virtual arrival policy in berth allocation. Transportation Science, 49:939-956, 2015.

L. A. G. Franzese, L. O. Abdenur, R. C. Botter, D. Starks, and A. R. Cano. Simulating the panama canal: present and future. In Proceedings of the 2004 winter simulation conference, volume 2, pages 1835-1838, 2011.

A. H. Gharehgozli, D. Roy, and R. de Koster. Sea container terminals: New technologies and OR models. Maritime Economics \& Logistics, 18(2):103-140, 2016.

M. Golias, I. Portal, D. Konur, E. Kaisar, and G. Kolomvos. Robust berth scheduling at marine container terminals via hierarchical optimization. Computers \& Operations Research, 41:412 - 422, 2014.

E. Gregoris, E. Barbaro, E. Morabito, G. Toscano, A. Donateo, D. Cesari, D. Contini, and A. Gambaro. Impact of maritime traffic on polycyclic aromatic hydrocarbons, metals and particulate matter in venice air. Environmental Science and Pollution Research, 23(7):6951-6959, 2016.

C. Kontovas and H. Psaraftis. Reduction of emissions along the maritime intermodal container chain: operational models and policies. Maritime Policy and Management, 38(4):451-469, 2011.

E. Lalla-Ruiz, X. Shi, and S. Voß. The waterway ship scheduling problem. Transportation Research Part D: Transport and Environment, 2016.

E. Lübbecke. On- and Offline Scheduling of Bidirectional Traffic. In A. Fink et al., editor, Operations Research Proceedings, 2016.

R.M. Lusby, J. Larsen, M. Ehrgott, and D. Ryan. Railway track allocation: Models and methods. OR Spectrum, 33(4):843-883, 2011.

D. Mavrakis and N. Kontinakis. A queueing model of maritime traffic in Bosporus Straits. Simulation Modelling Practice and Theory, 16:315-328, 2008.

R.M. Nauss. Optimal sequencing in the presence of setup times for tow/barge traffic through a river lock. European Journal of Operational Research, 187:1268-1281, 2008.

T.H. Nogueira, C.R.V. de Carvalho, G.P.A. Santos, and L.C. de Camargo. Mathematical model applied to singletrack line scheduling problem in brazilian railways. 4OR, 13(4):403-441, 2015.

K. E. Parnell, T. Soomere, L. Zaggia, A. Rodin, G. Lorenzetti, J. Rapaglia, and G. M. Scarpa. Ship-induced solitary riemann waves of depression in venice lagoon. Physics Letters A, 379(6):555 - 559, 2015.

W. Passchyn, S. Coene, D. Briskorn, J. L. Hurink, F. C.R. Spieksma, and G. Vanden Berghe. The lockmaster's problem. European Journal of Operational Research, 251(2):432 - 441, 2016. 
P. Pellegrini, G. Marliere, R. Pesenti, and J. Rodriguez. RECIFE-MILP: An Effective MILP-Based Heuristic for the Real-Time Railway Traffic Management Problem. IEEE Transactions on Intelligent Transportation Systems, 16(5):2609-2619, 2015.

E. R. Petersen and A. J. Taylor. An optimal scheduling system for the Welland Canal. Transportation Science, 22: 173-185, 1988. doi: 10.1287/trsc.22.3.173.

E. Quaglietta, P. Pellegrini, R.M.P. Goverde, T. Albrecht, B. Jaekel, G. Marlière, J. Rodriguez, T. Dollevoet, B. Ambrogio, D. Carcasole, M. Giaroli, and G. Nicholson. The ON-TIME real-time railway traffic management framework: A proof-of-concept using a scalable standardised data communication architecture. Transportation Research Part C: Emerging Technologies, 63:23-50, 2016.

L. Styhre, H. Winnes, J. Black, J. Lee, and H. Le-Griffin. Greenhouse gas emissions from ships in ports - Case studies in four continents. Transportation Research Part D: Transport and Environment, 54:212 - $224,2017$.

Ö. S. Ulusçu and T. Altıok. Waiting time approximation in single-class queueing systems with multiple types of interruptions: modeling congestion at waterways entrances. Annals of Operations Research, 172:291-313, 2009.

Ö. S. Ulusçu, B. Öbaş, T. Altıok, I. Or, and T. Yılmaz. Transit vessel scheduling in the Strait of Istanbul. The Journal of Navigation, 62:59-77, 2009.

J. Verstichel, P. De Causmaecker, F. Spieksma, and G. V. Berghe. The generalized lock scheduling problem: An exact approach. Transportation Research Part E: Logistics and Transportation Review, 65:16 - 34, $2014 \mathrm{a}$.

J. Verstichel, P. De Causmaecker, F.C.R. Spieksma, and G. Vanden Berghe. Exact and heuristic methods for placing ships in locks. European Journal of Operational Research, 235:387-398, 2014b.

X. Wang, M. J. Arnesen, K. Fagerholt, M. Gjestvang, and K. Thun. A two-phase heuristic for an in-port ship routing problem with tank allocation. Computers \& Operations Research, 91:37 - 47, 2018.

Z. Yang, H. Shi, K. Chen, and H. Bao. Optimization of container liner network on the Yangtze River. Maritime Policy \& Management, 41(1):79-96, 2014.

X. Zhang, J. Lin, Z. Guo, and T. Liu. Vessel transportation scheduling optimization based on channel-berth coordination. Ocean Engineering, 112:145 - 152, 2016.

L. Zhen, Z. Liang, D. Zhuge, L. H. Lee, and E. P. Chew. Daily berth planning in a tidal port with channel flow control. Transportation Research Part B: Methodological, 106:193 - 217, 2017. 